\title{
Telemetry Vibration Signal Trend Extraction Based on Multi-scale Least Square Algorithm \\ Feng GUO
}

PLA 91550 Unit 94, Liaoning Dalian, 116023, China

346502272@qq.com

Keywords: Telemetry; Trend Extraction; Multi-scale Least Square; EMD

\begin{abstract}
A new trend extraction method based on multi-scale least square algorithm combined with EMD was proposed for telemetry vibration signal. The observed signal is decomposed by EMD to obtain a series of IMF, based on which the least square algorithm is adopted to complete trend extraction with different fitting order. For telemetry vibration signal has non-stationary characteristic, the traditional trend extraction method cannot obtain ideal result. The fitting order serious affects the performance of least square algorithm for trend extraction which has no reasonable method of design. The multi-scale least square algorithm can take advantage of the EMD and the fitting order can be designed according to different IMF, which can improve the trend extraction precision effectively. The simulation and test data processing results show the effectiveness of the proposed method.
\end{abstract}

\section{Introduction}

Trend extraction and de-noising are two key technologies of telemetry environmental parameters during the pre-processing. The trend term in the telemetry environmental parameters is mainly due to the sensor affected by temperature, humidity and other external environment changes, which shows a trend of zero deviation, sometimes the trend also produced in the data receiving device for the existence of the system error [1]. Trend term does not has a priori information and uniform change, the only feature is that the trend term has long cycle which is greater than the length of the data record [2]. The trend term affects not only the time domain correlation analysis and the mean square value statistical result, but also the frequency spectrum analysis for there is a large component at the low frequency. The trend extraction methods mainly include average slope method, difference method, low pass filtering method and least square fitting method etc. These methods general need to deal with signal with a priori information and has a certain scope of application, such as average slope method and difference method are only can extract the linear trend [3]. Although low pass filtering method and least square fitting method can deal with polynomial and exponential type trend extraction [4], the filtering or fitting parameters setting are difficult, which results in the trend of the extraction of the integrity and the degree of loss of signal information is difficult to grasp. The least square fitting method has a good ability to extract polynomial trend and exponential trend if the fitting order is set reasonable. Hereby, combined with EMD, a multi-scale least square algorithm for trend extraction was proposed. The observed signal is decomposed by EMD to obtain a series of IMF, based on which the least square algorithm is adopted to complete trend extraction with different fitting order. The multi-scale least square algorithm can take advantage of the EMD and the fitting order can be designed according to different IMF, which can improve the trend extraction precision effectively. The simulation and test data processing results show the effectiveness of the proposed method.

\section{Trend Extraction by Least Square Algorithm}

Although the trend term is very complex and difficult to grasp the law, it can classify the trend and study its effective extraction methods. The trend usually can be divided into three types: linear trend, polynomial trend and exponential type trend, also a more complex trend can be obtained 
through the combination of these three trends. Three trends can be given by

$$
\begin{aligned}
& r(t)=a_{0}+a_{1} t \\
& r(t)=a_{0}+a_{1} t+a_{2} t^{2}+\cdots+a_{k} t^{k} \\
& r(t)=a e^{-b t}
\end{aligned}
$$

Where $a, b, a_{0}, a_{1} \cdots a_{k}$ are the relation parameters of the trend.

Least square fitting method has good ability to extract the polynomial trend and exponential type trend [5], however, the fitting order has a great influence on the extraction performance of the trend term, furthermore, the size of the fitting order cannot be determined in advance. The least square fitting order of experience is less than 5. If the size of the fitting order is too large, it is fitted to extract the trend of information is likely to contain a portion of the signal information, instead, if the fitting order is too small, for polynomial or exponential trend will produce extraction totally inadequate. If the polynomial can be expressed by $y(n)$ and the order of the polynomial is $K$, according to the principle of least square algorithm, $y(n)$ can be given by

$$
y(n)=\sum_{j=1}^{K} a_{j}(n \Delta T)^{j} \quad n=1,2, \cdots, N
$$

Where $\Delta T$ is the sampling time interval, $a_{j}$ is the coefficient of least square fitting polynomial. If let $y(n)$ approach the trend $r(n)$, minimum mean square error of $y(n)$ and $r(n)$ is expected as follow

$$
J(n)=\sum_{n=1}^{N}(r(n)-y(n))^{2}=\sum_{n=1}^{N}\left[r(n)-\sum_{j=0}^{K} a_{j}(n \Delta T)^{j}\right]^{2} \quad j=1,2, \cdots K
$$

Then the least square algorithm transforms to solve the extreme-value problem of $J(n)$.

$$
\frac{\partial J(n)}{\partial a_{j}}=2 \sum_{n=1}^{N}\left[r(n)-\sum_{j=0}^{K} a_{j}(n \Delta T)^{j}\right]\left[-(n \Delta T)^{j}\right]=0
$$

According to Eq.6, least square algorithm can obtain $K+1$ equation as follow

$$
\sum_{j=0}^{K} \sum_{n=1}^{N} a_{j}(n \Delta T)^{j}=\sum_{n=1}^{N} r(n)(n \Delta T)^{j}
$$

If the fitting order $K$ is determined, the coefficient $a_{j}$ can be solved and the trend can be extracted. If the trend term is linear, least square algorithm can set the fitting order is 1 . In a large number of engineering practices, the fitting order is set to 3 [6]. But so far, there is no theoretical basis for the determination of the polynomial fitting order, and it can only be set according to the specific processing conditions of the signal.

\section{Multi-scale Least Square Combined with EMD}

EMD is a non-stationary signal processing method proposed by N.E.Huang, which is an adaptive decomposition method of signal without basis function. The basic idea of EMD is to extract the signal into a series of IMF by means of the method of extracting the mean value of the maximum envelope and the iterative method. Compared with the wavelet transform, EMD no longer needs the bases function and set the scale parameter and the translation parameter, which has the self-adaptation. From the point of view of data processing, EMD provides a method to decompose complex signals into simple single component signals, which can refine the processing of data and get more ideal results.

EMD decomposition based on the following assumptions: (1) Any complex signal can be represented by a combination of a series of IMF; (2) The defect points in the data can be removed and obtained by using the method of differential, decomposition and integral to obtain IMF; (3) The characteristic time scale of the signal is determined by the time interval between the extreme points. During the process of the EMD, IMF is obtained according to the iterative operation of the mean 
value of the extreme envelope and the upper and lower envelope of the signal. Let the multi-component signal is $x(t)$, EMD find all the local maximum and local minimum points, based on which the upper and lower envelope can be obtained by three times of the interpolation. Let the mean of the upper and lower envelope is $m_{1}(t)$, then the difference of $m_{1}(t)$ and $m_{1}(t)$ is given by

$$
x(t)-m_{1}(t)=h_{1}(t)
$$

If $h_{1}(t)$ meets the condition of IMF, $h_{1}(t)$ is the first IMF of $x(t)$. However, $h_{1}(t)$ usually does not meet the IMF condition for the complex multi-component signal, especially for telemetry vibration signal. Let $h_{1}(t)$ is the new signal to decompose and the mean of the upper and lower envelope of $h_{1}(t)$ is $m_{11}(t)$, then the difference of $m_{11}(t)$ and $h_{1}(t)$ is

$$
h_{1}(t)-m_{11}(t)=h_{11}(t)
$$

If $h_{11}(t)$ is still does not meet the condition of IMF, repeat above iterative operator until $h_{1 k}(t)$ meets the condition of IMF. Let $c_{1}(t)=h_{1 k}(t)$ and $c_{1}(t)$ is the first IMF of $x(t)$. Remove $c_{1}(t)$ from $x(t)$ as follow

$$
x(t)-c_{1}(t)=r_{1}(t)
$$

Normally, $r_{1}(t)$ is still a multi-component signal, which can be taken as a new signal to carry on EMD iterative process as above until all the IMF has been separated from $x(t)$.

$$
r_{1}(t)-c_{2}(t)=r_{2}(t) ; \cdots ; r_{n-1}(t)-c_{n}(t)=r_{n}(t)
$$

Where $r_{n}(t)$ is the residual component which has long cycle compared with IMF, which is usually a monotonic function only have a maximum of extreme points.

According to the analysis of trend characteristics and the traditional trend extraction method, the priori information about the trend of the signal acquisition in engineering is generally not obtained in advance. Least squares fitting the trend term extraction method can meet the trend of various types of extraction. However, the fitting order of least square has a great influence on the result of trend term extraction. If the order is too small, least square algorithm for polynomial or exponential trend extraction may be incomplete. Instead, if the fitting order number is too large, the trend extraction often contains some useful signal information, which result loss of the useful information of the signal. Since the trend and the frequency of the signal is corresponding, a new method can be provide combined with EMD called multi-scale least square algorithm.

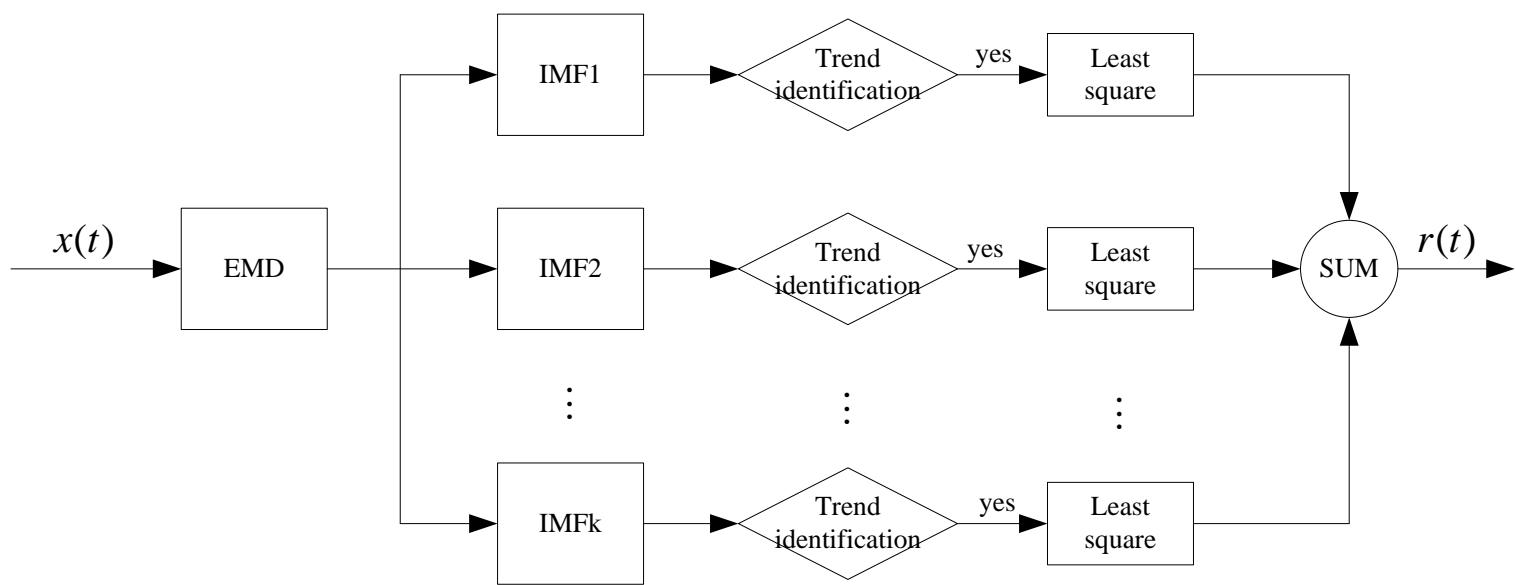

Fig.1 Multi-scale least square trend extraction process

Fig. 1 shows the multi-scale least square trend extraction process. The signal is decomposed by EMD to obtain a series IMF, and then according to the mean of the IMF to determine whether the IMF contains the trend term. If there is a trend in the IMF, the least square fitting order is set according to the scale or the order of the IMF, and the trend extraction is carried on the IMF. Finally, the last trend is obtained by sum all the trend terms which are extracted from the IMF. The advantage of multi-scale least square trend extraction is that the least square fitting operation carry 
on the IMF of EMD rather than the signal itself, the IMF which does not include trend term can be reserved directly, the other IMF which include trend term can set different fitting order according to the scale of IMF. Multi-scale least square trend extraction method can extract the trend with high precision under the premise of protecting the useful signal information to the maximum extent.

\section{Simulation and Data Processing}

To verify the validity of the multi-scale least square trend extraction method, simulation and test data is processed respectively. The simulation signal $x(t)$ is formed by the superposition of the sine signal and the sampling frequency is $f_{s}=1000 \mathrm{~Hz}$. Let $f_{k}$ is the center frequency and $b_{k}$ is the amplitude of the different sine signal and the simulation signal is given by

$$
x(t)=\sum_{k=1}^{K} b_{k} \sin \left(2 \pi f_{k} t\right)+r(t)
$$

The trend $r(t)$ is given by

$$
r(t)=a_{0}+a_{1} t+a_{2} t^{2}+a_{3} t^{3}
$$

Where $a_{0}=0.2, a_{1}=0.5, a_{2}=0.5$ and $a_{3}=0.01$. Fig. 2 shows the waveform of the simulation signal and Fig. 3 shows the waveform of the simulation trend.

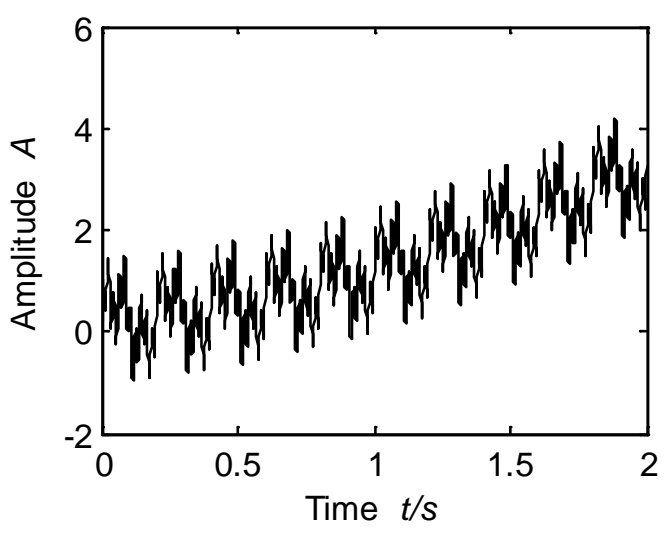

Fig.3 The simulation signal

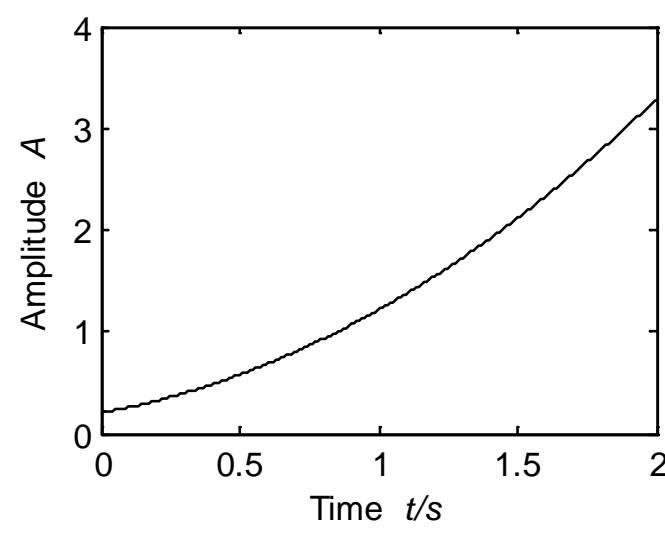

Fig.4 The simulation trend

Three trend extraction methods are carried on the simulation signal, the trend extraction result of multi-scale least square (EMD+LS) is shown in Fig.5. Residual of different methods is shown in Fig.6. Fig.6 shows that using the residual component as the trend (EMD-residual) and least square (LS) trend extraction has large residual error than EMD+LS, which shows the effectiveness of multi-scale least square extraction method.

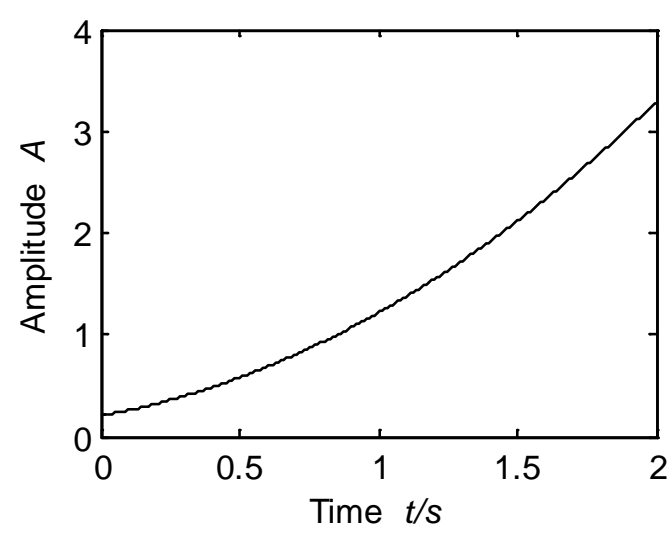

Fig.5 Trend extraction by multi-scale least square

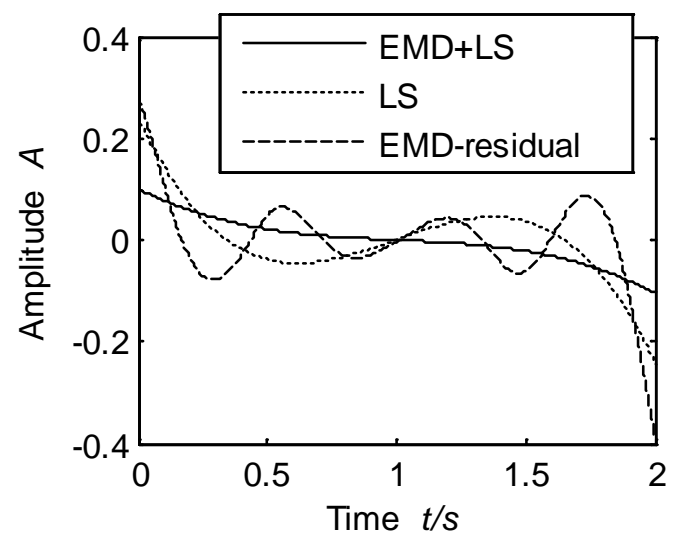

Fig.6 Residuals of different methods

To further prove the performance of multi-scale least square trend extraction method proposed in this paper, the high frequency vibration in one vehicle test is processed. The sampling frequency is 
5kHz. The waveform of the signal is shown in Fig.7. Fig.8 shows the trend term extracted by multi-scale least square algorithm. The trend result shows that the proposed method has good performance in telemetry vibration signal processing.

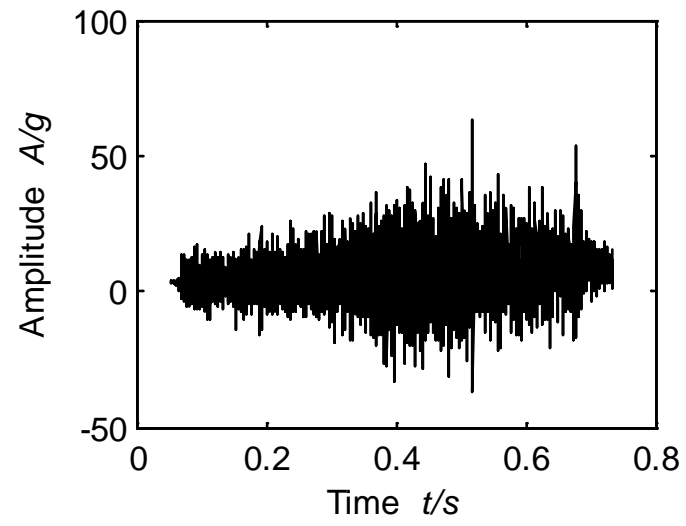

Fig.7 High frequency vibration signal

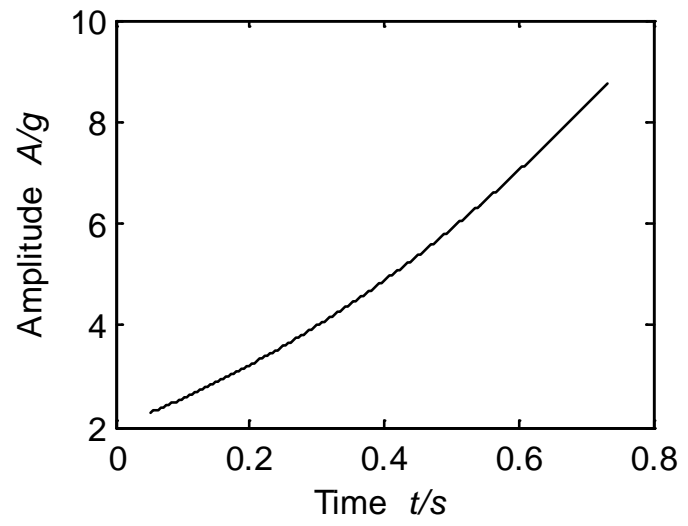

Fig.8 The trend extracted by EMD-LS

\section{Conclusion}

This work proposed a multi-scale least square trend extraction method combined with EMD. For the telemetry vibration signal has non-stationary characteristic and the trend has complex component, the performance of the traditional least square fitting method affected by the fitting order. Multi-scale least square trend extraction carries on trend extraction on the IMF rather than the signal, which can set different fitting order according to the scale of IMF and obtain trend extraction with high precision under the premise of protecting signal information from loss. The simulation and test data processing results show the effectiveness of the proposed method.

\section{References}

[1] YANG D., WANG X. T., XU G. L. Research on 1D signal fast trend extracting via multi-scale extrema [J]. Journal of Electronics \& Information Technology, 2013, 35(5): 2018-1214.

[2] QU C. S., XU H. L., TAN Y., et al. Trend extraction from laser gyro drift data based on modified empirical mode decomposition [J]. Journal of Astronautics, 2009, 30(2): 597-603.

[3] CAO Q., YUE D.J., GAO Y.P., et al. Contrast study on various methods extracting trend extraction based on non-stationary time series [J]. Journal of Geodesy and Geodynamics, 2013, 33(6): 150-154.

[4] CHNE J., XU Y.L. Application of EMD to signal trend extraction [J]. Journal of Vibration, Measurement \& Diagnosis, 2005, 25(2): 101-104+153.

[5] JIN X.J. Outliers elimination method of ballistic measurement data based on least square fitting [J]. Sichuan ARMAMENTARII, 2011, 1: 20-23.

[6] WU X.P., WANG F.M. The research of trend remove based on the principle of least-squares method [J]. Control \& Automation, 2008, 24(10-3): 254-255. 